\title{
HTS POWER LEADS FOR THE BTEV INTERACTION REGION*
}

\author{
S.Feher ${ }^{\#}$, R.Carcagno, D.Orris, T. Page, Y.Pischalnikov, R. Rabehl, C. Sylvester, M. Tartaglia, \\ J.C. Tompkins, Fermi National Accelerator Laboratory \\ Batavia, IL 60510, U.S.A.
}

\begin{abstract}
A new Interaction Region (IR) for the BTEV experiment was planned to be built at Fermilab. This IR would have required new superconducting quadrupole magnets and many additional power circuits for their operation. The new "low beta" quadrupole magnet design was based upon the Fermilab LHC quadrupole design, and would have operated at $9.56 \mathrm{kA}$ in $4.5 \mathrm{~K}$ liquid helium. The use of conventional power leads for these circuits would have required substantially more helium for cooling than is available from the cryogenic plant, which is already operating close to its limit. To decrease the heat load and helium cooling demands, the use of HTS power leads was necessary. In developing specifications for HTS leads for the BTEV interaction region, several 6 kA HTS leads produced by American Superconductor Corporation (ASC) have been tested at over-current conditions. Final design requirements were to be based on these test results. This paper summarizes the test results and describes the design requirements for the $9.65 \mathrm{kA}$ HTS power leads.
\end{abstract}

\section{INTRODUCTION}

The 10kA current leads for the high gradient quadrupoles in the BTEV IR at Fermilab were to be made from high temperature superconductor (HTS) to avoid additional loading of the $4.5^{\circ} \mathrm{K}$ He system. In the present Tevatron configuration, four spool pieces have been modified to incorporate 6 kA HTS leads [1]-[3], and one of these has been installed and operated in the ring for several years.

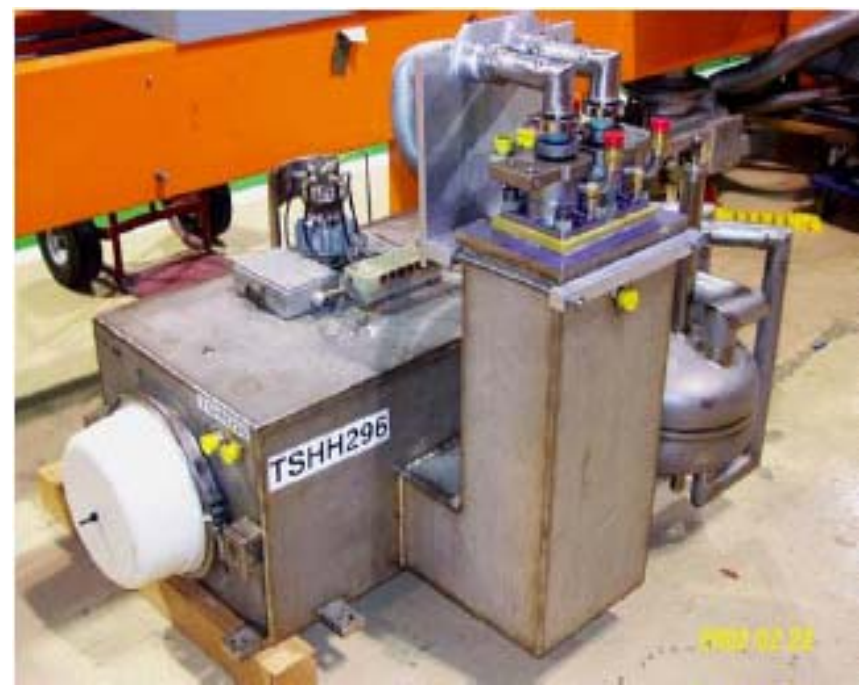

Figure 1. Standard Tevatron spool with HTS leads.
These HTS current leads made by American Superconductor Corporation consist of parallel tapes of BSCCO-2223 multifilamentary powder-in-tube conductor in a silver alloy matrix soldered to a conventional copper upper section. The vapor-cooled conventional copper section operates between room temperature and twophase nitrogen temperature, while the HTS section operates between this region and $4 \mathrm{~K}$. One of these modified spool pieces is shown in Figure 1. The HTS lead assembly and the LN2 reservoir are clearly visible in the foreground and right side of the picture, respectively.

\section{TEST RESULTS}

Based on R\&D tests performed during the $6 \mathrm{kA}$ lead program, it appeared that it could be possible to operate the leads at higher currents by increasing the coolant flow.

Preliminary tests of an existing HTS spool at the Magnet Test Facility (MTF) verified this hypothesis: it ran in a stable mode at the nominal operating current of 9.56 A. More detailed tests of a second pair of HTS leads have were carried out in a dewar facility which allowed greater control over cryogenic pressure, temperature, and flow. These tests were very successful [3]. Both the upper conventional copper section which is cooled with liquid nitrogen vapor and the lower HTS section cooled with liquid helium vapor exhibited stable operation up to a $10 \mathrm{kA}$ current and up to $200 \mathrm{~A} / \mathrm{s}$ current ramp rate.

To demonstrate that the lead is robust we performed a coolant loss test by closing the liquid nitrogen flow valve and waiting for the quench detection system to detect the abnormal situation and to ramp down the power supply. Both the HTS and copper section voltages rose, but the copper section was the first to pass the threshold set by the manufacturer. We did not observe any irregular behaviour of the leads during operation following the coolant-loss test.

The successful high current performance of the first two lead pairs tested demonstrated that a single pair of leads of the existing design will be sufficient for operation at $10 \mathrm{kA}$. The spool design was modified, reducing the leads required to one pair from the original two pair configuration. Testing of additional lead pairs was continued both to further confirm these results and to qualify the remaining leads in the spares pool.

A third pair of ASC HTS power leads was tested at MTF in February 2005. The test was to again confirm the stable operation at $10 \mathrm{kA}$. This goal was achieved by operating the leads for $\sim 3$ hours steadily (see Figure 2). In total, three pairs of leads have been tested in two 
different test configurations: one pair in a spool and two pair in a dewar. All of them exhibited excellent performance at the desired 9.5-10 kA current range.

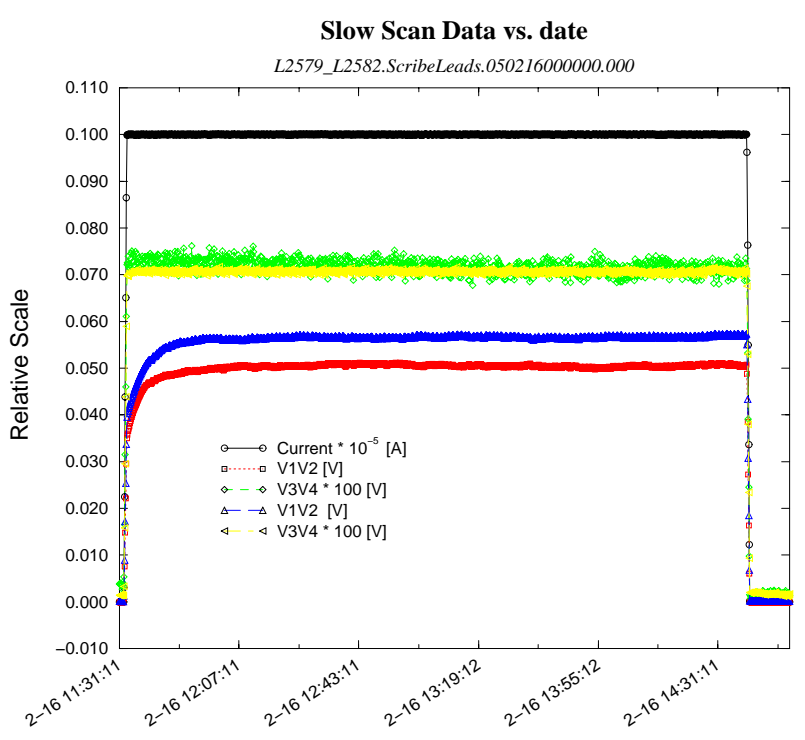

Figure 2. DC Stability test. Current and lead voltages (arbitrary scale) indicating stable operation at 10kA for about 3 hours. V1V2 signals correspond to the voltage segments attached to the copper sections while V3V4 correspond to the HTS sections.

The ASC power leads were originally designed for Tevatron operations so the low temperature superconductor (LTS) part of the leads consists of Tevatron type cable which is not as wide as the bus planned for the BTEV magnets. Splicing the Tevatron cable to the new bus posed an additional challenge. At the lower section of the HTS power leads, between the copper block and the HTS tapes, a relatively low temperature solder ( 140 C meting point) was used. To make the joint between the LTS part of the lead and the LTS bus for the magnets, we had to make sure that the solder temperature was kept low enough not to un-solder the adjacent HTS-LTS joint. A special test setup was prepared and the temperature was monitored during the soldering process using a standard $\mathrm{PbSn}$ solder. The temperature just above the solder joint was kept under $140 \mathrm{C}$ for the entire soldering process. This new joint configuration was also tested in February. A satisfactory low splice resistance was achieved as evidenced by the measurement shown in Figure 3.

To avoid frosting of the upper end of the leads when excessive cooling is applied, a special thermal insulation scheme was applied onto the outer surface of the leads. First the entire top section of the lead was coated with Conothane CE 1155 Polyurethane Prepolymer (Cytec Industries Inc.). After this coat cured, a layer of Si-Coat 570 High Voltage Insulating Coating (CLS Silicones Inc) was applied to prevent development of water channels. Two-component polyurethane spray foam (Handi Foam;
Fomo Product Inc.) followed the silicon coat mainly to thermally insulate the lead. The foam was also coated with silicon so any open channel was sealed from moisture.

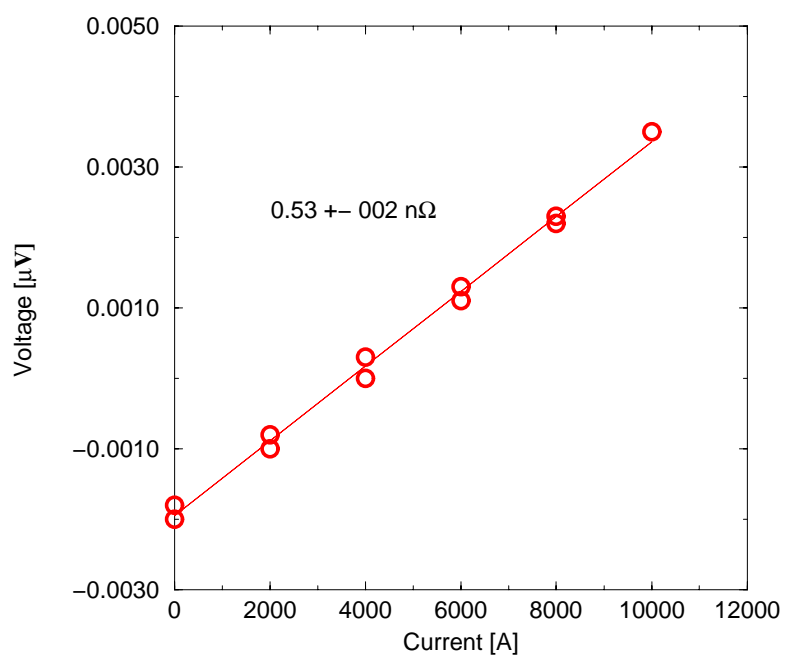

Figure 3. Splice voltage $(\mu \mathrm{V})$ versus lead current $(\mathrm{A})$. The splice resistance determined from these measurements, $0.5 \mathrm{n} \Omega$, was satisfactory.

This insulation scheme worked very well. During the test the cold side of the insulation was close to $-60 \mathrm{C}$ and no frost was observed (see Figure 4).

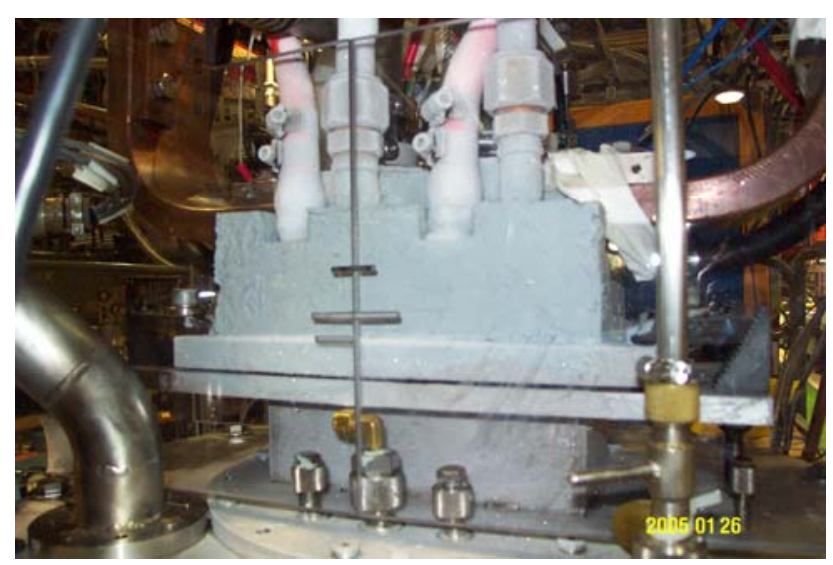

Figure 4. Thermal insulation scheme applied to the leads is shown. Although the vapor gas outlets are cold and consequently accumulation frost can be observed the foam insulation remained dry.

The results of the tests of three different pairs of HTS leads are consistent. After these tests we were confident that the Tevatron lead design was sufficient for $10 \mathrm{kA}$ use and that we could have proceeded with qualifying the remains spares and procure the additional leads required for the project. 


\section{DESIGN REQUIREMENTS}

Based on the test results described in the previous section we concluded that most of the requirements developed for the ASC $6 \mathrm{kA}$ power leads were appropriate for the new design. Consequently the focus of the new requirements was to list the design changes relative to those $6 \mathrm{kA}$ ASC power lead design which were incorporated into the Tevatron spools:

1. The voltage tap connector insulation scheme was improved*.

2. Spacing between the positive and negative leads was increased from 4 in to 5 in. This change made it easier to deal with the electrical separation of the leads and not to increase unnecessarily the stray magnetic field.

3. Improved electrical insulation between the housing of the spool and the support plate of the lead was introduced**.

4. Power flag connection to the $10 \mathrm{kA}$ bus had to be redesigned to accommodate the $60 \%$ increase in power. This flag cooling/warming with water had to be redesigned as well but the power lead flag remained the same.

5. To prevent frosting due to increased cooling conditions at the top part of the lead assembly special insulation scheme was developed.

6. Soldering material and technique had to be modified to increase the solder joint strength.

The conceptual design of the new spool containing HTS power leads is shown in Figure 5. The power leads are positioned toward the inner radius of the tunnel to avoid interference with cryogenic pipes and external high current busses along the outer wall. A large opening was included at the bottom section of the satellite chimneys where the leads are placed to allow free flow of liquid helium. This satellite structure also made it possible to control the liquid level for the power leads independently from the main helium bath.

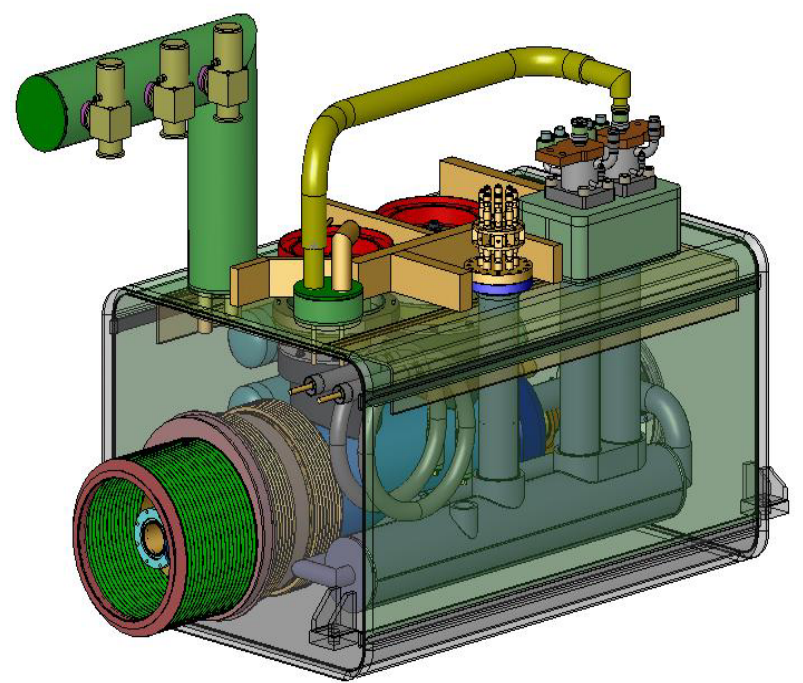

Figure 5. Conceptual design of the new spool containing HTS power leads.

\section{CONCLUSIONS}

In total, three pairs of ASC made HTS power leads have been tested - one pair in a spool and two in a dewar - in two different test configurations; all of them exhibited excellent performance at the desired 9.5-10 kA current range.

The HTS power lead design requirements for the C0 IR are almost identical with the $6 \mathrm{kA}$ ASC design requirements with only few minor exceptions: improved connector and power lead electrical isolation from the spool box, improved thermal insulation of the top of the lead assembly, modified LTS to LTS soldering technique and increased spacing between the power leads.

\section{REFERENCES}

[1] G. Citver, “HTS Power Lead Test Results”, PAC'99 New York, April 1999, pp. 1420.

[2] J. Brandt et al., "Operating Experience with HTS Leads at the Tevatron", Advances in Cryogenic Engineering,, American Institute of Physics, New York, 2002, pp.567-574.

[3] S. Feher et al., "Tevatron HTS Power Lead Test", FERMILAB-CONF-04-257-TD

\footnotetext{
* The connector for voltage tap signals was not properly prepared by ASC and consequently High Voltage stand off value of the lead was compromised. The base of the connector where the wires are exposed to helium must be embedded into epoxy.

** Surface of the G10 insulating plate between the housing of the spool and the support plate of the lead had a fine epoxy finish to be able to seal the lead with metal C-seals. With time this type of design solution tends to fail and leaks can occur. To obtain a more conservative and reliable solution thin layer of Stainless Steel was added on both sides of the plate. One side of the SS "washer" which is glued with stycast to the G10 plate should have a rough surface and the other side should have the desired fine finish which complies with the standard C-seal requirements.
} 
